OPEN ACCESS

Edited by:

Pedro M. Baptista,

University of Zaragoza, Spain

Reviewed by:

Daniela Calina,

University of Medicine and Pharmacy

of Craiova, Romania

Kunkai Su,

Zhejiang University, China

${ }^{*}$ Correspondence:

Xiaojie Huang

huangxiaojie78@126.com

tThese authors have contributed equally to this work

Specialty section:

This article was submitted to Gastroenterology,

a section of the journal

Frontiers in Medicine

Received: 19 May 2020

Accepted: 21 July 2020

Published: 07 August 2020

Citation:

Gao Y, Li Q, Shi H, Feng Y, Zhang T,

Chen Y, Liang L, Chen D, Wu H, Jin R and Huang $X$ (2020) Preliminary Exploration of the Cause of Liver Disorders During Early Stages in COVID-19 Patients.

Front. Med. 7:501.

doi: 10.3389/fmed.2020.00501

\section{Preliminary Exploration of the Cause of Liver Disorders During Early Stages in COVID-19 Patients}

\author{
Yuan $\mathrm{Gao}^{1 \dagger}$, Qi $\mathrm{Li}^{2 \dagger}$, Hongbo Shi ${ }^{3+}$, Yingmei Feng ${ }^{4}$, Tong Zhang ${ }^{5}$, Yu Chen ${ }^{1}$, \\ Lianchun Liang ${ }^{6}$, Dexi Chen ${ }^{3}$, Hao $\mathrm{Wu}^{5}$, Ronghua $\mathrm{Jin}^{3}$ and Xiaojie Huang ${ }^{5 *}$ \\ 1 Difficult \& Complicated Liver Diseases and Artificial Liver Center, Beijing Youan Hospital, Capital Medical University, Beijing, \\ China, ${ }^{2}$ Department of Hepatology and Gastroenterology, Beijing Youan Hospital, Capital Medical University, Beijing, China, \\ ${ }^{3}$ Beijing Youan Hospital, Beijing Institute of Hepatology, Capital Medical University, Beijing, China, ${ }^{4}$ Center for Clinical \\ Research, Beijing Youan Hospital, Capital Medical University, Beijing, China, ${ }^{5}$ Center for Infectious Diseases, Beijing Youan \\ Hospital, Capital Medical University, Beijing, China, ${ }^{6}$ Department of Infectious Disease, Beijing Youan Hospital, Capital \\ Medical University, Beijing, China
}

Background: Abnormal liver function is a common indication of coronavirus disease 2019 (COVID-19) patients. Two proposed mechanisms are liver injury mediated by angiotensin-converting enzyme 2 (ACE2) and the involvement of the systemic immune response. We investigated the role played by these to determine the cause of liver abnormality in the early stages of COVID-19.

Methods: A cross-sectional study was conducted among confirmed cases of COVID-19 at Beijing Youan Hospital from January 21, 2020, to February 24, 2020. We compared clinical characteristics, viremia status, and cytokine profile on admission between patients with and without liver disorder.

Results: Of the 44 COVID-19 patients analyzed, there were no differences in the clinical symptoms and signs, disease severity, or computed tomography (CT) image features between the two groups. Lymphopenia was more common in the liver disorder group. Further, C-reactive protein levels were much higher in the hepatic disorder group, with significantly higher concentrations of IL-6, IL-10, and M-CSF. Viremia was detected in only $7 \%$ of patients.

Conclusions: Due to the infrequency of viremia, ACE2-mediated viral hepatitis does not seem to account for the commonly observed liver disorders in COVID-19 patients. By contrast, a dysregulated immune response may be a crucial pathogenic factor for liver disorder in the early stages of COVID-19.

Keywords: COVID-19, immune response, liver injury, early stage, cytokines

\section{INTRODUCTION}

In the largest-scale study to date of the clinical characteristics of coronavirus disease 2019 (COVID-19) patients in China, about one-fifth of cases featured elevated alanine aminotransferase (ALT) levels on admission, with a higher prevalence in critically ill patients (1). The mechanism underlying this abnormal liver function remains a matter of debate. Because angiotensin-converting enzyme 2 (ACE2) has been confirmed to be the receptor for the entry of 
TABLE 1 | Demographics and baseline characteristics of patients with COVID-19.

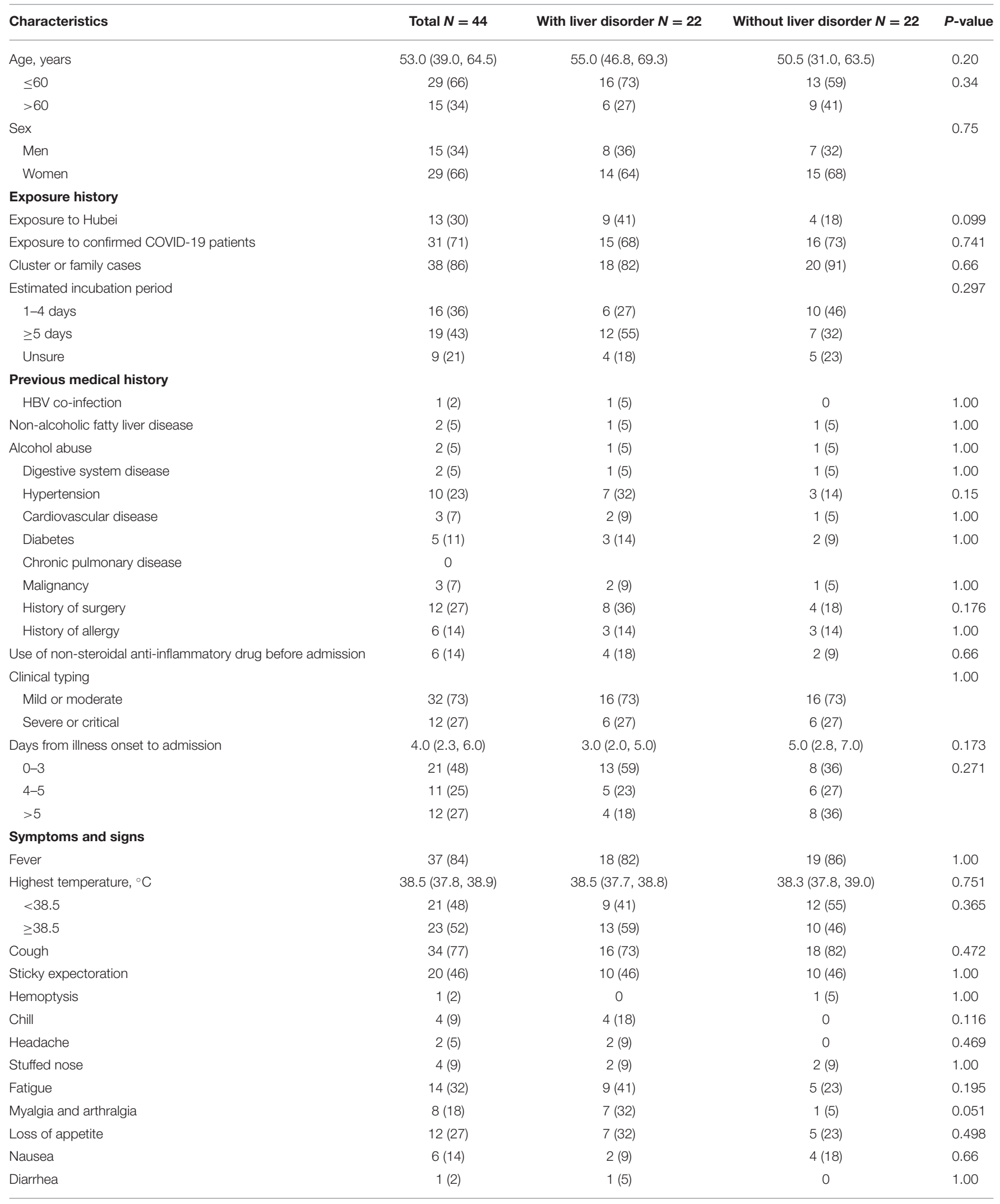


TABLE 1 | Continued

\begin{tabular}{|c|c|c|c|c|}
\hline Characteristics & Total $N=44$ & With liver disorder $N=22$ & Without liver disorder $N=22$ & $P$-value \\
\hline Shortness of breath & $18(41)$ & $8(36)$ & $10(46)$ & 0.54 \\
\hline Systolic pressure, mmHg & $120.0(112.0,130.0)$ & $120.5(108.0,140.5)$ & $120.0(112.8,130.0)$ & 0.75 \\
\hline Respiratory rate $>24$ breaths/min & $1(2)$ & $1(5)$ & 0 & 1.00 \\
\hline Arterial oxygen pressure $<60 \mathrm{mmHg}$ & $8(18)$ & $4(18)$ & $4(18)$ & 1.00 \\
\hline CT findings & & & & 0.41 \\
\hline Normal or unilateral pneumonia & $7(16)$ & $2(9)$ & $5(23)$ & \\
\hline Bilateral diffused ground-glass opacity & $37(84)$ & $20(91)$ & $17(77)$ & \\
\hline
\end{tabular}

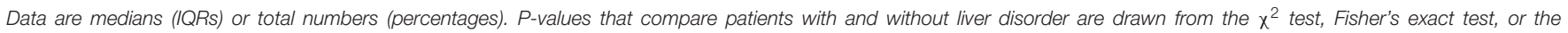
Mann-Whitney U-test.

severe acute respiratory syndrome coronavirus 2 (SARS-CoV-2) into the cells (2) and is expressed in multiple organs and tissues (3), such as cholangiocytes, which are involved in many aspects of liver physiology, including regeneration and adaptive immune response mechanisms, and dysfunction of cholangiocytes can cause hepatocytes damage, it has been proposed that the virus could cause direct extrapulmonary-organ dysfunction, such as liver injury. But it is important to note that an elevation of cholestatic markers is not found among all COVID-19 patients with liver disorders (4). It is also possible that hepatic damage relates to the systemic immune response $(5,6)$. After SARSCoV-2 invades airway epithelial cells, partial patients show aggressive inflammatory responses. The vast release of cytokines by the immune system in response to the viral infection could result in a cytokine storm, and compromise multiple organs, including the liver (7). To clarify which factor plays the most crucial role in liver disorders during the early stages of COVID-19 and provide scientific evidence to aid physicians' decision-making in clinical practice, we conducted a crosssectional study to compare viremia status and cytokine levels between COVID-19 patients with and without liver disorder on admission.

\section{PATIENTS AND METHODS}

From January 21, 2020, to February 24, 2020, a total of 92 patients with COVID-19, confirmed by real-time RT-PCR tests of nasopharyngeal swabs, were admitted to Beijing Youan Hospital in China. Written informed consent was obtained from 44 patients who were enrolled in the study, among whom 22 had liver disorder, defined as abnormal plasma levels of ALT exceeding $40 \mathrm{U} / \mathrm{L}$ in women or $50 \mathrm{U} / \mathrm{L}$ in men (Siemens advia2400, Germany), regardless of AST level.

Epidemiological, demographic, clinical, laboratory, and radiological data were extracted from the patients' electronic medical records.

Plasma samples drawn from all subjects at admission were stored in the biobank of Beijing Youan Hospital. The levels of the cytokines and chemokines IL- $1 \alpha$, IL-1 $\beta$, IL-1RA, IL3, IL-5, IL-6, IL-7, IL-8 (also known as CXCL8), IL-9, IL-10, IL-12p40, IL-12p70, IL-13, IL-15, IL-17A, eotaxin (also known as CCL11), FGF2, G-CSF (CSF3), M-CSF, IFN- $\alpha 2$, MCP-1
(CCL2), MIP-1 $\alpha$ (CCL3), MIP-1 $\beta$ (CCL4), TNF- $\alpha$, TNF- $\beta$, and VEGF-A in plasma taken on admission were analyzed using the Luminex 200TM assay (Millipore, Billerica, USA), according to the manufacturer's instructions.

A $200 \mu \mathrm{L}$ plasma sample from each patient was analyzed using a Duplex Real-time PCR Diagnostic Kit for Rapid Detection of 2019-nCoV ORF1ab/N gene (A7712RC-50T, XABT, Beijing, China). Viremia was defined as a positive result for real-time RT-PCR in the plasma sample.

\section{Study Approval}

The study protocol was approved by the Ethics Committee for Beijing Youan Hospital, Capital Medical University.

\section{Statistical Analyses}

Continuous variables are expressed as medians (IQRs) and were compared using the Mann-Whitney $U$-test. Categorical variables are expressed as numbers (percentages) and were compared using the $\chi^{2}$ test or Fisher's exact test. All comparisons were between patients with and without liver disorders. Boxplots are given to describe the concentrations of cytokines and chemokines in plasma. Two-sided $\alpha<0.05$ were considered to indicate statistical significance. Statistical analyses were performed using IBM SPSS Statistics software version 21.0 and Graphpad Prism version 8.0.2.

\section{RESULTS}

Before hospitalization, four of 22 patients with and two of 22 patients without liver disorders were temporarily treated with a non-steroidal anti-inflammatory drug. The median time from symptom onset to hospitalization was four (IQR 2.3-6.0) days. On admission, the median level of ALT in the liver disorder group was $58.5 \mathrm{U} / \mathrm{L}$, with one value in excess of $200 \mathrm{U} / \mathrm{L}$ (264 $\mathrm{U} / \mathrm{L})$, but no patient had overt bilirubinemia. No significant differences were seen between the two groups in the onset of symptoms, disease severity, or CT image features (Table 1). Most laboratory tests did not present a distinction between two groups. Lymphopenia was found in 17 (77\%) of 22 patients with liver disorder and eight (36\%) of 22 patients without liver disorder $(p=0.006)$ (Table 2). The median level of C-reactive protein (CRP) was higher in the hepatic disorder group, with 
TABLE 2 | Laboratory results for patients with COVID-19 on admission to the hospital.

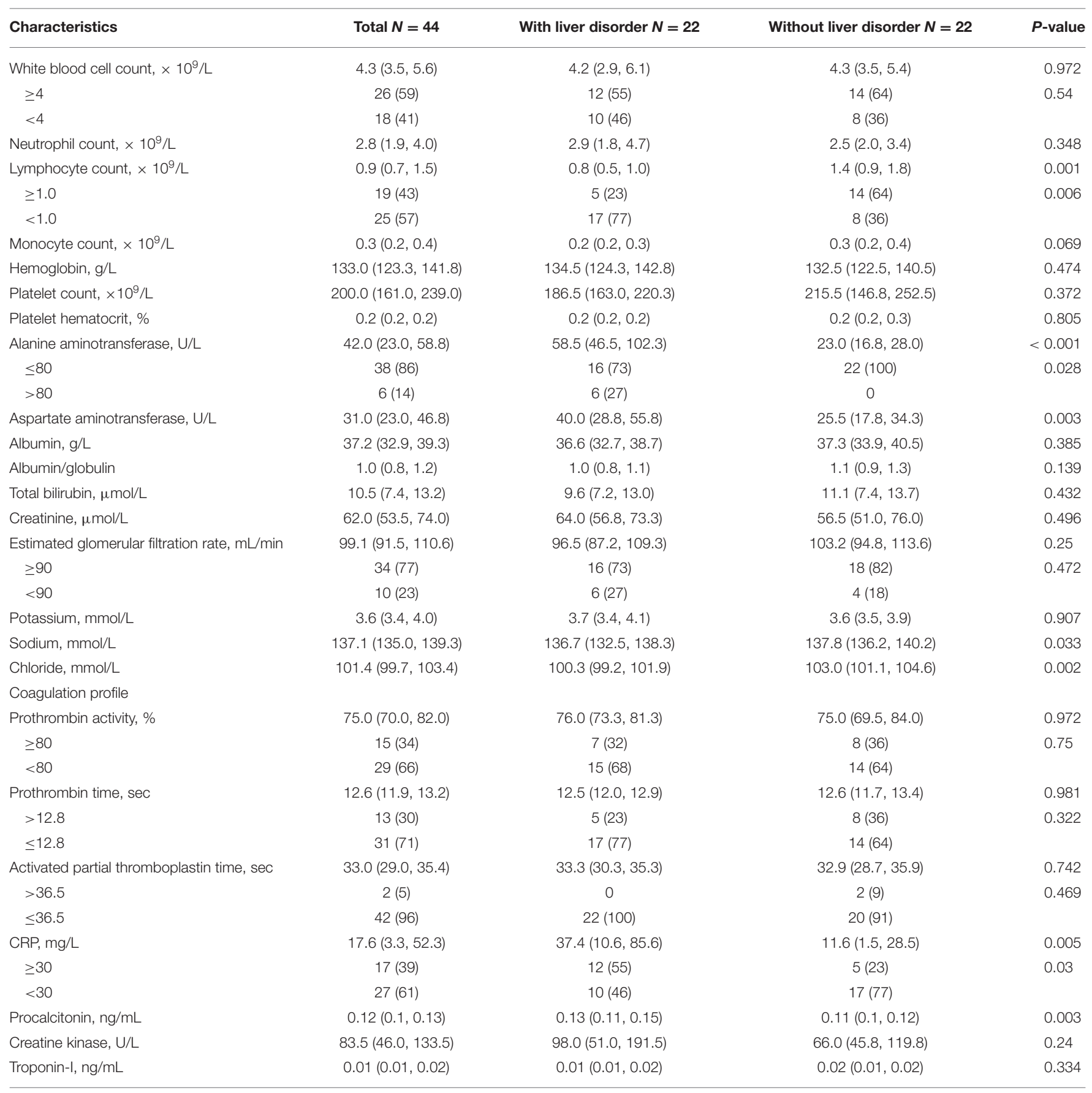

Data are medians (IQRs) or total numbers (percentages). P-values that compare patients with and without liver disorder are from the $\chi^{2}$ test, Fisher's exact test, or the Mann-Whitney U-test.

a significantly higher concentration of IL-6, IL-10, and MCSF (Figure 1). All measured cytokines and chemokines are presented in detail in Figure S1. Excluding three patients with missing plasma samples, viremia was only detected in three (7\%) of 41 patients, that is, in two patients with liver disorder and in one without.

\section{DISCUSSION}

Although our study did not reveal any dynamic change or prognosis during the clinical course, as a cross-sectional study, we found that there is no relationship between liver condition and hospital medication. 


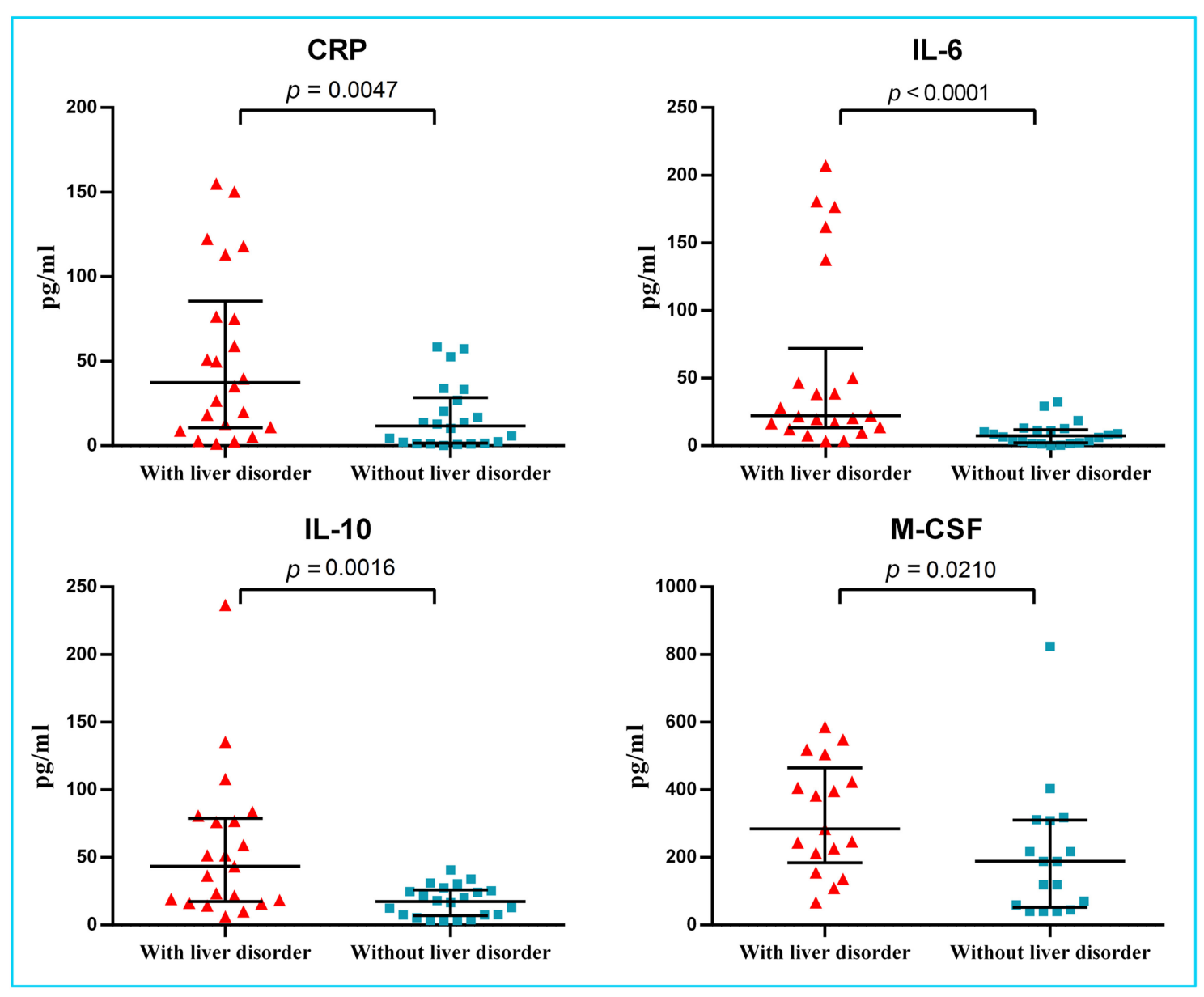

FIGURE 1 | Levels of CRP and several cytokines in plasma of COVID-19 patients with and without liver disorder. Significantly increased CRP levels and higher levels of IL-6, IL-10, and M-CSF were found in COVID-19 patients with liver disorder $(n=22)$ than those without liver disorder $(n=22)$. CRP, C-reactive protein; IL, interleukin; M-CSF, macrophage colony-stimulating factor.

Previous investigations have indicated that the incidence of viremia in COVID-19 patients ranges from 8 to $15 \%(8,9)$. Our study also found viremia to be uncommon in patients with and those without liver disorder on admission. If viremia is required for SARS-CoV-2 to cause direct liver injury via the ACE2 receptor, SARS-CoV-2 hepatitis could not account for the commonly observed liver disorders in COVID-19 patients. A recent review of seven studies suggested that elevated ALT and AST may not necessarily be hepatogenic (10), as similar liver abnormalities are also thought to be common in other respiratory viral infections. Despite the large number of COVID-19 patients with liver injury, there have been no reports of liver failure caused by this disease (11). From the point of pathology, SARS-CoV2 RNA has been detected in liver tissue from some fatal cases (12), but other autopsies of COVID-19 patients have not revealed the typical pathological manifestation of viral hepatitis caused by SARS-CoV-2 $(13,14)$.

However, our findings support the possibility that a dysregulated immune response may be a crucial pathogenic factor in liver disorders. On admission, the levels of CRP and the production of the cytokines IL-6, IL-10, and M-CSF were significantly elevated in patients with a liver disorder. The early study suggested that, with ensuing local inflammation of lung affected areas, secretion of the pro-inflammatory cytokines and chemokines was increasing, such as IL-6, IFN $\gamma, \mathrm{M}-\mathrm{CSF}$, and MCP1, and these would release into the blood of COVID19 patients (9). In severe patients, levels of IL-2, IL-7, IL10, granulocyte colony-stimulating factor (G-CSF), and IP-10 elevated more significantly than in mild or moderate patients. A markedly higher levels of IL- 6 and IL-10 related with a progression of disease severity and poor prognosis (15). Unlike previous reports $(16,17)$, we didn't identify the difference of IL$1 \beta$, an important cytokine released during pyroptosis, probably due to the early stage of a cross-sectional study. Notably, such derailed non-specific cytokine secretion could easily result in liver disorders, which is common in other infectious disease (18).

In our study, overt bilirubinemia was absent in COVID19 patients with elevated ALT levels. The overproduction of these inflammation-related cytokines with mild liver function abnormality do not seem to be a typical pattern in known 
viral, alcohol-, or drug-induced hepatitis. For other coexisting conditions that could influence liver, the four patients in the liver disorder group used non-steroidal anti-inflammatory drug (NSAID) and two patients without liver disorder used NSAID; other patients' liver function did not have a problem with drug interference at admission. The patients with respiratory distress or arterial oxygen pressure $<60 \mathrm{mmHg}$ were evenly distributed in each group. There were no patients with multiple organ dysfunction at admission. Thus, the commonly observed liver disorders cannot be attributed to the above causes. Taken together, these indications lead to the conclusion that the observed liver disorder is more likely a secondary injury of an aberrant immune response, as has been shown for other human coronavirus infections (19).

Our study had several limitations. First, the viremia was checked qualitatively, not quantitatively, but due to its rarity, a qualitative test is not unacceptable for viremia detection. Second, we did not test GGT or ALP, so most patients had no ultrasonic records if they had not declared a history of liver diseases. After obtaining a medical history through inquiries, it appeared possible that fatty liver, chronic cholecystitis, or even compensatory cirrhosis might have been present in some cases but was not diagnosed. Third, except for the remarkable change in pro-inflammatory cytokines, functional exhaustion and reduced diversity of $\mathrm{T}$ cells is another immune dysfunction profile in COVID-19 patients, but has not been included in the current study (20). Finally, we recognize the small size of the sample; the available data are insufficient to distinguish enough types of cytokines' differences between two groups. We nevertheless suggested that our clinical design might be useful for investigating whether SARS-CoV-2 directly targets other specific extrapulmonary organs (21).

In conclusion, our study provides evidence that liver disorders in COVID-19 patients results from a systemic immune response, but there may not be direct virus-induced hepatic cell damage. It should be kept in mind that liver disorders, even during the early course of COVID-19, indicate the occurrence of a drastic cytokine release status, which may be a sign of the possible fierce inflammation condition and rapid disease progression.

\section{DATA AVAILABILITY STATEMENT}

The datasets presented in this study can be found in online repositories. The names of the repository/repositories and accession number(s) can be found in the article/ Supplementary Material.

\section{REFERENCES}

1. Guan WJ, Ni ZY, Hu Y, Liang WH, Ou CQ, He JX, et al. Clinical characteristics of coronavirus disease 2019 in China. N Engl J Med. (2020) 382:170820. doi: 10.1056/NEJMoa2002032

2. Zhou P, Yang XL, Wang XG, Hu B, Zhang L, Zhang W, et al. A pneumonia outbreak associated with a new coronavirus of probable bat origin. Nature. (2020) 579:270-3. doi: 10.1038/s41586-020-2012-7

\section{ETHICS STATEMENT}

The studies involving human participants were reviewed and approved by Beijing Youan Hospital, Capital Medical University Ethical Committee. Written informed consent to participate in this study was provided by the participants' legal guardian/next of kin. Written informed consent was obtained from the individual(s), and minor(s)' legal guardian/next of kin, for the publication of any potentially identifiable images or data included in this article.

\section{AUTHOR CONTRIBUTIONS}

YG, QL, YF, YC, HW, RJ, and XH contributed to the study design, patient recruitment, data collection, data statistical analysis, data interpretation, literature search, and writing of the manuscript. $\mathrm{XH}, \mathrm{TZ}$, and LL contributed to the accuracy of the data analyses. HS and DC contributed to the laboratory tests and analyses. All authors reviewed and approved the final version of the manuscript.

\section{FUNDING}

This work was supported by grants from the National Megaprojects for Infectious Diseases in China (2017ZX10201101, 2020YFC0841700, 2020YFC0844900), Beijing Excellent Talent Plan (2018000021223ZK04), and Beijing Youan Hospital project for COVID-19 (BJYAYY-2020PY-03).

\section{ACKNOWLEDGMENTS}

We acknowledge all health care workers involved in the diagnosis and treatment of patients in Beijing Youan Hospital and we thank Professors Hui Chen (School of Biomedical Engineering, Capital Medical University, Beijing, China) and Junjie Xu (NHC Key Laboratory of AIDS Immunology, Department of Laboratory Medicine, the First Affiliated Hospital of China Medical University, Shenyang, China) for their guidance in the data analyses. Special thanks go to Professor Bing Hou (Xenorm MedInfo Center, Beijing, China) for the important suggestions given for the revision of this paper.

\section{SUPPLEMENTARY MATERIAL}

The Supplementary Material for this article can be found online at: https://www.frontiersin.org/articles/10.3389/fmed. 2020.00501/full\#supplementary-material

3. Zou X, Chen K, Zou J, Han P, Hao J, Han Z. Single-cell RNAseq data analysis on the receptor ACE2 expression reveals the potential risk of different human organs vulnerable to 2019-nCoV infection. Front Med. (2020) 14:185-92. doi: 10.1007/s11684-02 0-0754-0

4. Xu L, Liu J, Lu M, Yang D, Zheng X. Liver injury during highly pathogenic human coronavirus infections. Liver Int. (2020) 40:9981004. doi: 10.1111/liv.14435 
5. Mehta P, McAuley DF, Brown M, Sanchez E, Tattersall RS, Manson JJ. COVID19: consider cytokine storm syndromes and immunosuppression. Lancet. (2020) 395:1033-4. doi: 10.1016/S0140-6736(20)30628-0

6. Tisoncik JR, Korth MJ, Simmons CP, Farrar J, Martin TR, Katze MG. Into the eye of the cytokine storm. Microbiol Mol Biol Rev. (2012) 76:1632. doi: 10.1128/MMBR.05015-11

7. Tay MZ, Poh CM, Rénia L, MacAry PA, Ng LFP. The trinity of COVID19: immunity, inflammation and intervention. Nat Rev Immunol. (2020) 20:363-74. doi: 10.1038/s41577-020-0311-8

8. Young BE, Ong SWX, Kalimuddin S, Low JG, Tan SY, Loh J, et al. Epidemiologic features and clinical course of patients infected with SARSCoV-2 in Singapore. JAMA. (2020) 323:1488-94. doi: 10.1001/jama.2020.3204

9. Huang C, Wang Y, Li X, Ren L, Zhao J, Hu Y, et al. Clinical features of patients infected with 2019 novel coronavirus in Wuhan, China. Lancet. (2020) 395:497-506. doi: 10.1016/S0140-6736(20)30183-5

10. Bangash MN, Patel J, Parekh D. COVID-19 and the liver: little cause for concern. Lancet Gastroenterol Hepatol. (2020) 5:529-30. doi: 10.1016/S2468-1253(20)30084-4

11. Tian D, Ye Q. Hepatic complications of COVID-19 and its treatment. J Med Virol. (2020). doi: 10.1002/jmv.26036. [Epub ahead of print].

12. Puelles VG, Lütgehetmann M, Lindenmeyer MT, Sperhake JP, Wong MN, Allweiss L, et al. Multiorgan and renal tropism of SARS-CoV-2. N Engl J Med. (2020). doi: 10.1056/NEJMc2011400. [Epub ahead of print].

13. Xu Z, Shi L, Wang Y, Zhang J, Huang L, Zhang C, et al. Pathological findings of COVID-19 associated with acute respiratory distress syndrome. Lancet Respir Med. (2020) 8:420-2. doi: 10.1016/S2213-2600(20)30076-X

14. Yao XH, Li TY, He ZC, Ping YF, Liu HW, Yu SC, et al. [A pathological report of three COVID-19 cases by minimal invasive autopsies]. Zhonghua Bing Li Xue Za Zhi. (2020) 49:411-7. doi: 10.3760/cma.j.cn112151-20200312-00193

15. Chen G, Wu D, Guo W, et al. Clinical and immunological features of severe and moderate coronavirus disease 2019. J Clin Invest. (2020) 130:26209. doi: 10.1172/JCI137244

16. Cavalli G, De Luca G, Campochiaro C, Della-Torre E, Ripa M, Canetti D, et al. Interleukin-1 blockade with high-dose anakinra in patients with COVID-19, acute respiratory distress syndrome, and hyperinflammation: a retrospective cohort study. Lancet Rheumatol. (2020) 2:e325-31. doi: 10.1016/S2665-9913(20) 30127-2

17. Alijotas-Reig J, Esteve-Valverde E, Belizna C, Selva-O'Callaghan A, PardosGea J, Quintana A, et al. Immunomodulatory therapy for the management of severe COVID-19. Beyond the anti-viral therapy: A comprehensive review. Autoimmun Rev. (2020) 19:102569. doi: 10.1016/j.autrev.2020. 102569

18. Yan J, Li S, Li S. The role of the liver in sepsis. Int Rev Immunol. (2014) 33:498-510. doi: 10.3109/08830185.2014.889129

19. Channappanavar R, Perlman S. Pathogenic human coronavirus infections: causes and consequences of cytokine storm and immunopathology. Semin Immunopathol. (2017) 39:529-39. doi: 10.1007/s00281-01 7-0629-X

20. Zheng HY, Zhang M, Yang CX, et al. Elevated exhaustion levels and reduced functional diversity of $\mathrm{T}$ cells in peripheral blood may predict severe progression in COVID-19 patients. Cell Mol Immunol. (2020) 17:5413. doi: 10.1038/s41423-020-0401-3

21. Li H, Liu L, Zhang D, Xu J, Dai H, Tang N, et al. SARS-CoV-2 and viral sepsis: observations and hypotheses. Lancet. (2020) 395:151720. doi: 10.1016/S0140-6736(20)30920-X

Conflict of Interest: The authors declare that the research was conducted in the absence of any commercial or financial relationships that could be construed as a potential conflict of interest.

Copyright (C) 2020 Gao, Li, Shi, Feng, Zhang, Chen, Liang, Chen, Wu, Jin and Huang. This is an open-access article distributed under the terms of the Creative Commons Attribution License (CC BY). The use, distribution or reproduction in other forums is permitted, provided the original author(s) and the copyright owner(s) are credited and that the original publication in this journal is cited, in accordance with accepted academic practice. No use, distribution or reproduction is permitted which does not comply with these terms. 